\title{
ENSEMBLE MONTE CARLO STUDY OF ELECTRON TRANSPORT IN BULK INDIUM NITRIDE
}

\author{
E. Bellotti *, B. Doshi ${ }^{*}$, K. F. Brennan * and P. P. Ruden ${ }^{* *}$ \\ *School of ECE, Georgia Tech, Atlanta, GA 30332, kbrennan@ece.gatech.edu \\ ** Dept. of ECE, University of Minnesota, Minneapolis, MN 55455
}

Cite this article as: MRS Internet J. Nitride Semicond. Res. 4S1, G21511

\begin{abstract}
Ensemble Monte Carlo calculations of electron transport at high applied electric field strengths in bulk, wurtzite phase InN are presented. The calculations are performed using a full band Monte Carlo simulation that includes a pseudopotential band structure, all of the relevant phonon scattering agents, and numerically derived impact ionization transition rates. The full details of the first five conduction bands, which extend in energy to about $8 \mathrm{eV}$ above the conduction band minimum, are included in the simulation. The electron initiated impact ionization coefficients and quantum yield are calculated using the full band Monte Carlo model. Comparison is made to previous calculations for bulk $\mathrm{GaN}$ and $\mathrm{ZnS}$. It is found that owing to the narrower band gap in $\mathrm{InN}$, a lower breakdown field exists than in either $\mathrm{GaN}$ or $\mathrm{ZnS}$.
\end{abstract}

\section{INTRODUCTION}

Wide band-gap semiconductors are becoming of increasing importance in many emerging optoelectronic and electronic device applications. Among these applications are ultraviolet (UV) photodetectors, blue and UV light emitters, and high frequency, high power electronic devices. Of the emerging wide band-gap semiconductors, the most promising candidates for power field effect transistors, FETs, are $\mathrm{SiC}$ and the III-nitrides. It is well known that $\mathrm{SiC}$ or $\mathrm{GaN}$ based transistors offer significantly higher maximum output power than comparable structures made from GaAs or $\mathrm{Si}[1,2]$. Owing to their relatively wide and direct energy band-gap, the III-nitride semiconductors are in addition particularly useful for UV and blue-light photonic detectors and emitters. The III-nitrides offer an additional advantage since heterostructures can be made from these materials. Along with $\mathrm{GaN}$, the InN ternary alloy, InGaN, has found application in a variety of heterostructure based opto-electronic devices. In spite of its potential application, little information is available about the transport properties of InGaN or its constituent binary materials, InN and GaN. Some progress has been made on GaN [3-10], but only a single limited study of electron transport in InN [11] has yet been performed.

It is the purpose of this paper to present the first theoretical study of the high field electronic transport properties of bulk InN. The calculations are performed using a full band, ensemble Monte Carlo simulation that includes a numerical formulation of the interband impact ionization transition rate [12]. The electron initiated impact ionization coefficients are calculated as a function of applied electric field strength. 


\section{MODEL DESCRIPTION}

The band structure of InN used within the Monte Carlo simulation is calculated using the empirical pseudopotential method. Though ab initio methods have been applied to the study of $\mathrm{InN}$ [13], the empirical pseudopotential method is employed herein since it offers a computationally efficient and reasonably accurate accounting of the band structure. The band structure derived from the pseudopotential model is shown in Figure1.

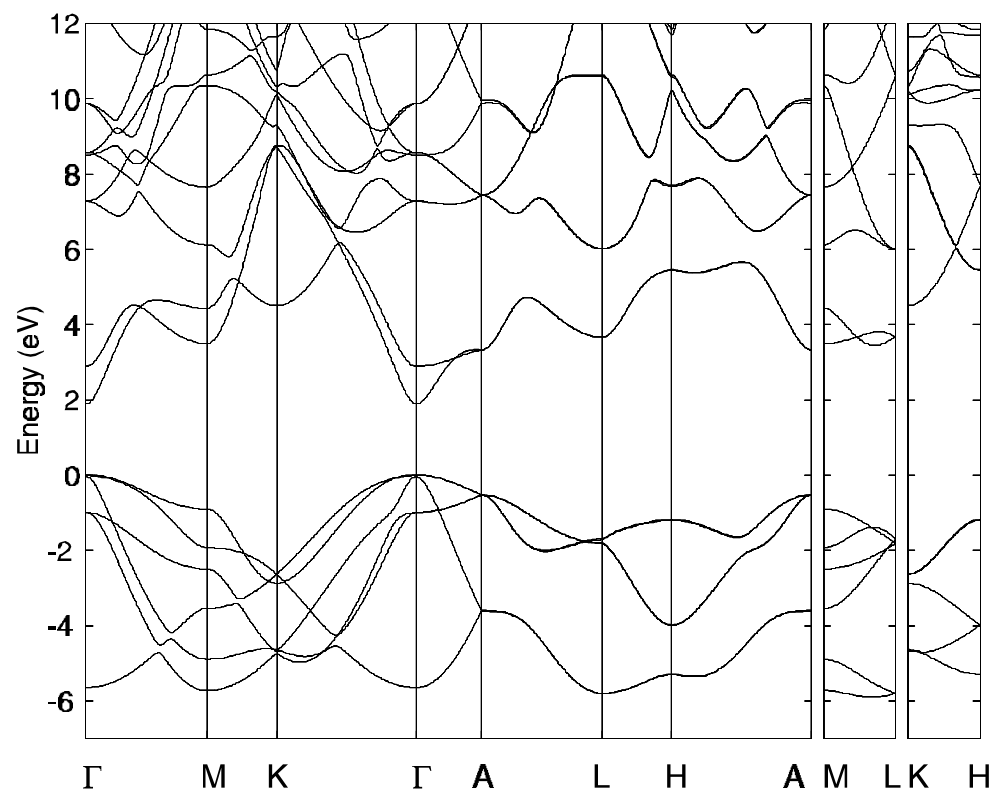

Fig. 1 Calculated pseudopotential band-structure of wurtzite $\mathrm{InN}$

Electron-phonon and impurity scattering form the other principal input into the Monte Carlo model. Polar-optical, acoustic phonon, ionized impurity, and piezoelectric scattering are included for electron energies below $0.9 \mathrm{eV}$. The acoustic phonon scattering is formulated inelastically. Above $0.9 \mathrm{eV}$, only polar optical, deformation potential and impact ionization scatterings are included. This choice is made to avoid using intervalley scattering explicitly since the intervalley deformation potentials are unknown. By substituting a general deformation potential scattering mechanism in place of the many intervalley mechanisms, the parametrization can be greatly reduced. Therefore, only one isotropic deformation potential scattering mechanism based on the realistic density of states is employed at electron energies greater than $0.9 \mathrm{eV}$. The coupling constant for this deformation potential scattering is determined by matching the low energy and high energy rates at $0.9 \mathrm{eV}$. The material parameters used to calculate the scattering rates are collected in Table I. The interband impact ionization transition rate is computed numerically from the pseudopotential band structure using a wave-vector dependent dielectric function following the approach of Kolnik et al. [12]. The wavevector dependent rate is computed on a 924 point grid for the first five conduction bands within the irreducible wedge of the first Brilloiun zone. The total transition rate is then determined by averaging the wave-vector dependent rate over constant energy surfaces. 
Table I. Bulk material parameters used in the phonon scattering rate calculations for wurtzite type InN.

\begin{tabular}{cc}
\hline \hline Parameter & Value \\
\hline Lattice constant $(\AA)$ [14] & $\mathrm{a}=3.54 \mathrm{c}=5.7$ \\
Low frequency dielectric constant [14] & 15.4 \\
High frequency dielectric constant [14] & 8.4 \\
Energy band gap (eV) [15] & 1.86 \\
Longitudinal Sound velocity $(\mathrm{cm} / \mathrm{s})[14]$ & $6.2410^{5}$ \\
Transverse Sound velocity $(\mathrm{cm} / \mathrm{s})[14]$ & $2.5510^{5}$ \\
Density $\left(\mathrm{g} \mathrm{cm}^{-3}\right)[14]$ & 6.81 \\
Effective mass at $\Gamma\left(\mathrm{m}^{*} / \mathrm{m}_{0}\right)[15]$ & 0.11 \\
Nonparabolicity at $\Gamma\left(\mathrm{eV}^{-1}\right)[15]$ & 0.419 \\
Intravalley acoustic deformation potential $(\mathrm{eV})[14]$ & 7.1 \\
Optical phonon energy at $\Gamma(\mathrm{eV})[14]$ & 0.089 \\
Piezoelectric coupling constant $\mathrm{K}_{\mathrm{av}}^{2}[16]$ & 0.0652 \\
\hline \hline
\end{tabular}

\section{CALCULATED RESULTS}

The ionization coefficients are calculated using the Monte Carlo simulator. It is well known that the ionization coefficients depend strongly on the high energy phonon scattering rate. Unfortunately, little information about the high energy scattering rates is known even for the most studied semiconductors. The high energy electron-phonon scattering rate is dominated by deformation potential scattering. Deformation potential scattering is, to lowest order, isotropic and proportional to the final density of states. The deformation potential scattering rate is generally calculated assuming a single, constant, energy independent coupling constant whose value is ascertained by comparison of the Monte Carlo coefficients to experimental data. In the present situation, no experimental information about the ionization coefficients exists so no comparison to experimental data is possible. Instead, we select a baseline value for the deformation potential constant such that the high and low energy scattering rates match at $0.9 \mathrm{eV}$ as mentioned above. By varying the deformation potential constant from this baseline value, the sensitivity of the ionization coefficients to the choice of the scattering rate can be assessed. 
The calculated ionization coefficients for fields applied along the (110) and (001) directions in bulk $\mathrm{InN}$ are plotted in Figure 2. The solid curves shown in Figure 2 correspond to the calculated coefficients using the baseline phonon scattering rate.

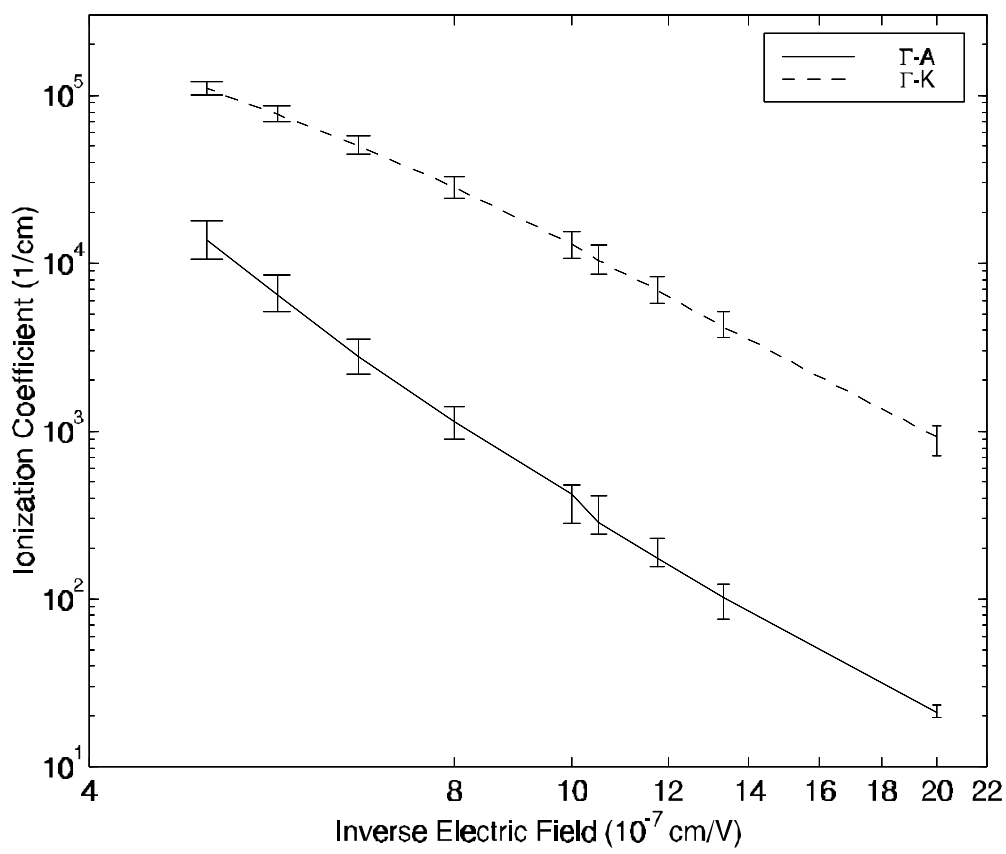

Fig.2 Calculated impact ionization coefficients for wurtzite $\mathrm{InN}$

There exists a substantial anisotropy in the ionization coefficients. Notice that the ionization coefficients are significantly lower for an applied field along the (001) direction than along the (110) direction. Since the present model assumes an isotropic high energy phonon scattering rate, the anisotropy in the ionization coefficients most likely is due to differences in the band structure along these two directions. Inspection of the band structure shows that the first two conduction bands are well separated from the third and higher bands except near the K point. For fields applied along the (110) direction, the electrons can readily drift to states from which they can be scattered into the third and higher conduction bands. The ionization transition rate is substantially larger within the third and higher conduction bands than within the first or second bands. Therefore, when an electron transfers into the third or higher bands, it is highly likely to undergo an impact ionization event. Conversely, when the field is applied along the (001) direction, the electrons cannot directly drift to states from which they can transfer into the third and higher conduction bands. Given the large separation of the energy bands along this direction far fewer electrons, on average, then will transfer into the third and higher bands. As a result, the ionization coefficients are substantially lower for an applied field along the (001) direction.

The top end of the error bars shown in Fig.2 represents the variation of the ionization coefficient for a 5\% decrease in the high energy scattering rate. The bottom end of the error bars represents the variation of the ionization coefficient for a $5 \%$ increase in the high energy scattering rate. The total variation of the ionization coefficient is of the order of $\sim 25 \%$ in the (110) direction and $\sim 45 \%$ in the (001) direction. As is 
generally observed, the ionization coefficient is highly sensitive to variations in the scattering rate.

It is useful to further characterize the relative hardness of the threshold energy. A hard threshold implies that upon reaching the minimum energy for which an ionization event occurs, a carrier soon suffers an impact ionization event. Most semiconductor materials seem to exhibit relatively soft thresholds, i.e., the carriers drift to energies substantially above the minimum necessary for an ionization event before impact ionizing. The character of the threshold can best be ascertained from the quantum yield. The quantum yield is defined as the average number of impact ionization events caused by a high energy injected carrier until its kinetic energy relaxes below the ionization threshold through scattering and/or impact ionization. The calculated quantum yield for electrons in InN is shown in Figure 3. As can be seen from Fig. 3, a substantial number of carriers survive to energies of $5.5-6 \mathrm{eV}$, nearly three times the energy gap, before suffering an impact ionization event. This is an indication of a relatively soft threshold, significantly softer than that of either GaN [7] or $\mathrm{ZnS}^{17}$.

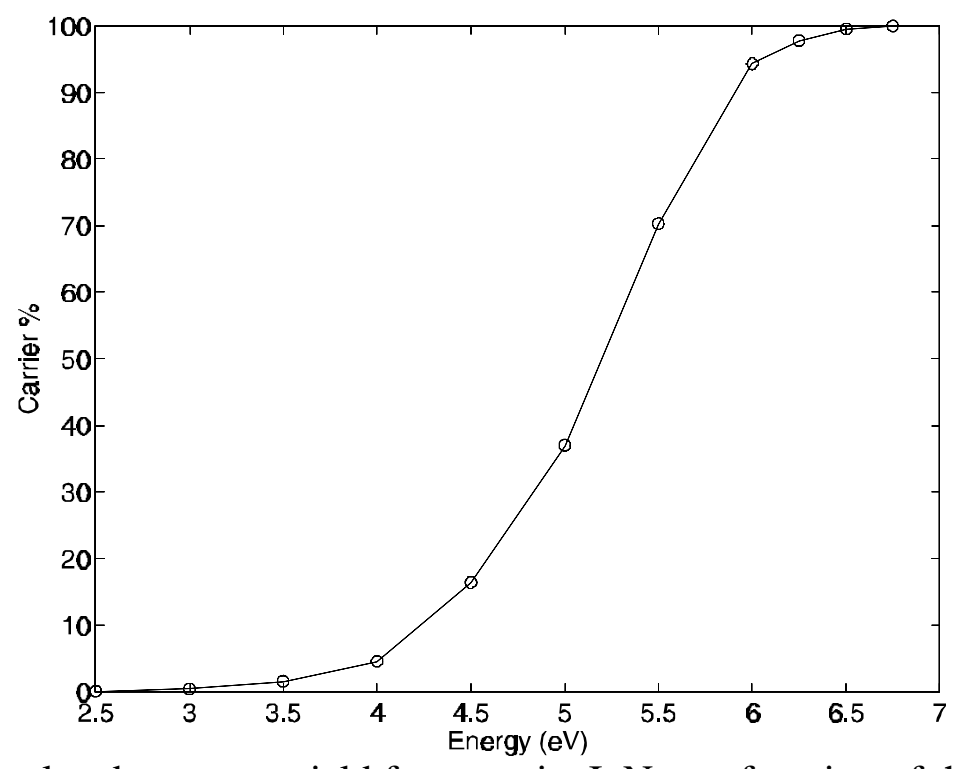

Fig.3 Calculated quantum yield for wurtzite $\mathrm{InN}$ as a function of the energy.

\section{CONCLUSIONS}

This paper presents the first study of high field electron transport in bulk, wurtzite InN. Theoretical results based on a full band, ensemble Monte Carlo model are presented. It is found that there exists a substantial anisotropy in the ionization coefficients, with the rate for fields applied along the (001) direction significantly less than for the (110) direction. It is further found that the threshold is relatively soft, significantly softer than either $\mathrm{GaN}$ or $\mathrm{ZnS}$.

\section{ACKNOWLEDGEMENTS}

This work was sponsored in part through a subcontract to Georgia Tech and the University of Minnesota from the Office of Naval Research MURI program at UCSB and 
by the National Science Foundation through a joint contract to the University of Minnesota and Georgia Tech, (ECS-9811366). The work at Georgia Tech was also supported by NORTEL through grant E21-5D7, and by the Office of Naval Research through contract E21-K19.

\section{References}

${ }^{1}$ C. Weitzel, L. Pond, K. Moore, and M. Bhatnagar, Proc. Int. Conf. on Silicon Carbide, III-Nitrides and Related Materials, Aug 31- Sept. 5, 1997, Stockholm, Sweden.

${ }^{2}$ K. Shenai, R. S. Scott, and B. J. Baliga, IEEE Trans. Electron Dev. 36, 1811 (1989).

${ }^{3}$ M. A. Littlejohn, J. R. Hauser, and T. H. Glisson, Appl. Phys. Lett. 26, 625 (1975).

${ }^{4}$ B. Gelmont, K. Kim, and M. Shur, J. Appl. Phys. 74, 1818 (1993).

${ }^{5}$ N. S. Mansour, K. W. Kim, and M. A. Littlejohn, J. Appl. Phys. 77, 2834 (1995).

${ }^{6}$ J. Kolnik, I. H. Oguzman, K. F. Brennan, R. Wang, P. P. Ruden, and Y. Wang, J. Appl. Phys. 78, 1033 (1995).

${ }^{7}$ J. Kolnik, I. H. Oguzman, K. F. Brennan, R. Wang, P. P. Ruden, J. Appl. Phys. 81, 726 (1997).

${ }^{8}$ I. H. Oguzman, J. Kolnik, K. F. Brennan, R. Wang, T.-N. Fang, and P. P. Ruden, J. Appl. Phys. 80, 4429 (1996).

${ }^{9}$ I. H. Oguzman, E. Bellotti, J. Kolnik, K. F. Brennan, R. Wang, and P. P. Ruden, J. Appl. Phys. 81, 7827 (1997).

${ }^{10}$ J. D. Albrecht, R. P. Wang, P. P. Ruden, M. Farahmand, and K. F. Brennan, J. Appl. Phys. 83, 4777 (1998).

${ }^{11}$ S. K. O'Leary, B. K. Foutz, M. S. Shur, U. V. Bapkar, and L. F. Eastman, J. Appl. Phys. 83, 826 (1998).

${ }^{12}$ J. Kolnik, I. H. Oguzman, K. F. Brennan, R. Wang, and P. P. Ruden, J. Appl. Phys. 79, 8838 (1996).

${ }^{13}$ W. R. L. Lambrecht and B. Segall, Properties of the Group III Nitrides, edited by J. E. Edgar (INSPEC, IEE, London, 1994).

${ }^{14}$ V. W. Chin, T. L. Tansley, and T. Osotchan, J. Appl. Phys. 75, 7365 (1994).

${ }^{15}$ Y.C. Yeo, T. C. Chong, and M.F. Li, J. Appl. Phys. 83, 1429 (1998).

${ }^{16}$ B. K. Ridley, Quantum Processes in Semiconductors, (Oxford, Oxford Univ. Press, 1982) p.123.

${ }^{17}$ E. Bellotti, K. F. Brennan, R. Wang, and P. P. Ruden, J. Appl. Phys. 83, 4765 (1998). 